\title{
Comparative Evaluation of Mechanical Properties of Cention N with Conventionally used Restorative Materials- An In Vitro Study
}

\author{
${ }^{1}$ Abhishek Mishra, ${ }^{2}$ Gautam Singh, ${ }^{3}$ Santosh K Singh, ${ }^{4}$ Manish Agarwal, ${ }^{5}$ Rizwan Qureshi, ${ }^{6}$ Nishant Khurana
}

\begin{abstract}
Objective: This study compared the compressive strength and flexural strength of Centurion $\mathrm{N}$ with other conventionally used restorative materials.

Materials and methods: Cention N, Amalgam, Glass lonomer Cement and Hybrid composite resin was used for sample fabrication. A total of 80 specimens were prepared. Forty samples $(\mathrm{n}=10$ each) were prepared for compressive strength and other 40 samples ( $n=10$ each) were prepared for flexural strength using aluminum split molds. The samples were tested using a universal Instron testing machine (UTM). Data were statistically analyzed using the Games-Howell post hoc test to determine whether statistically significant differences $(p<0.05)$ existed among the various restorative materials.
\end{abstract}

Results: Compressive strength and flexural strength of composites was significantly higher than cention N, GIC, and amalgam. Compressive strength of cention $\mathrm{N}$ was significantly higher than GIC. Flexural strength of cention $\mathrm{N}$ was found to be significantly higher than GIC and Amalgam.

Conclusion: Within the limitations of this study, it can be concluded that composite had the highest compressive strength and flexural strength of the four materials tested. However, cention $\mathrm{N}$ can be used in various restorative procedures in daily dental practice as a basic filling material along with tooth matching ability, it has good comparable mechanical properties and unlike composite, it's economical to patients.

Keywords: Amalgam, Cention N, Compressive strength, Flexural strength, Glass lonomer Cement, Hybrid composite.

How to cite this article: Mishra A, Singh G, Singh SK, Agarwal M, Qureshi R, Khurana N. Comparative Evaluation of Mechanical Properties of Cention N with Conventionally used Restorative

\footnotetext{
${ }^{1}$ Postgraduate Student, ${ }^{2}$ Assistant Professor, ${ }^{3}$ Reader, ${ }^{4}$ Professor, ${ }^{5,6}$ Lecturer

1,3,4,6 Department of Conservative Dentistry and Endodontics, Peoples College of Dental Sciences and Research Centre, Bhopal, Madhya Pradesh, India

${ }^{2}$ Department of Conservative Dental Sciences, IBN Sina National College, Jeddah, Makkah, Kingdom of Saudi Arabia

${ }^{5}$ Department of Conservative Dentistry and Endodontics, Bhaba Dental College and Research Centre, Bhopal, Madhya Pradesh, India
}

Corresponding Author: Abhishek Mishra, Postgraduate Student, Department of Conservative Dentistry and Endodontics, Peoples College of Dental Sciences and Research Centre, Bhopal, Madhya Pradesh, India, e-mail: mishra0904abhishek@ gmail.com
Materials-An In Vitro Study. Int J Prosthodont Restor Dent 2018;8(4):120-124.

Source of support: Nil

Conflict of interest: None

\section{INTRODUCTION}

Dental caries damaged tooth enamel and dentin. A carious tooth has an impaired shape and function. If attended early, it can be recovered and restored by the restorative material. A restorative material is one which re-establishes the biologic, functional and esthetic properties of healthy tooth structure. ${ }^{1}$ Numerous direct filling materials are available to the modern dental practice from amalgams to modern bulk fill composites. ${ }^{2}$ For the longevity of a restorative material, many factors play an important role. Strength is one of the important criteria. ${ }^{3}$ A restorative material should provide enough tensile and compressive strength to resist multidirectional masticatory forces for many years. ${ }^{4}$

Taking into account most widely used restorative materials, each has their advantages and disadvantage, and therefore a thorough knowledge of them will help the clinician in selection of appropriate material for a particular clinical situation. ${ }^{5}$

An amalgam is an alloy that contains mercury as one of its constituents. ${ }^{6}$ Amalgams were first introduced to western dentistry in the 19 th century. ${ }^{2}$ In practice, dental amalgam is a technique-insensitive material, and its long-term clinical performance is exceptional. Anusavice reports that approximately $90 \%$ of Amalgam restorations are still functional after 10 years. ${ }^{6}$ However, its relatively high coefficient of thermal expansion, the need for matrix band during condensation, the unaesthetic appearance and the Amalgam debate surrounding the safety of mercury has led to the development of tooth-colored restorative materials. The demand for tooth-colored restorations has grown considerably during the last decade. ${ }^{7}$

Resin-based composites (RBCs), ceramics and glass ionomer cement are tooth-colored restorative materials, to name a few. Nowadays, at least half of posterior direct restoration placements are done using resin- 
based composite restorative materials. The reasons for their worldwide popularity are their excellent esthetic value, needing minimal tooth preparation, providing bonding with the tooth structure and good retention. The advent of new composite restorative materials, together with new adhesives has brought enormous benefits-notably in terms of aesthetics and strides towards minimally invasive dentistry. They may, however, are perceived as expensive, time-consuming and technique sensitive. ${ }^{8}$

The glass ionomer cements developed by Wilson and Kent have several advantages such as fluoride release, adhesion to mineralized dental tissues and a coefficient of thermal expansion similar to that of tooth structure. ${ }^{9}$ In spite of so many favorable properties, its poor mechanical properties, limited indication range (unsuitable for stress bearing situations) and low esthetic value led to the further development of resin-based composites. ${ }^{10}$

Due to reasons mentioned abosve dentists have long sought a real alternative to silver amalgam, glass ionomer cement and composites, a cost-effective, fluoride releasing product that is quick and easy to use without complicated equipment and that offers both strength and good esthetics. ${ }^{11}$

Cention $\mathrm{N}$ is a new basic filling material developed offering these characteristics plus other advantages over both amalgams and glass ionomer cement.

Cention $\mathrm{N}$ is an "alkasite" restorative material which like compomer or ormocer is essentially a subgroup of the composite material class. This new category utilizes an alkaline filler, capable of releasing acid-neutralizing ions. ${ }^{11}$

The purpose of this study was to compare the compressive and flexural strength of cention $\mathrm{N}$ and other restorative materials to choose a appropriate restorative material for the restoration of a defective tooth.

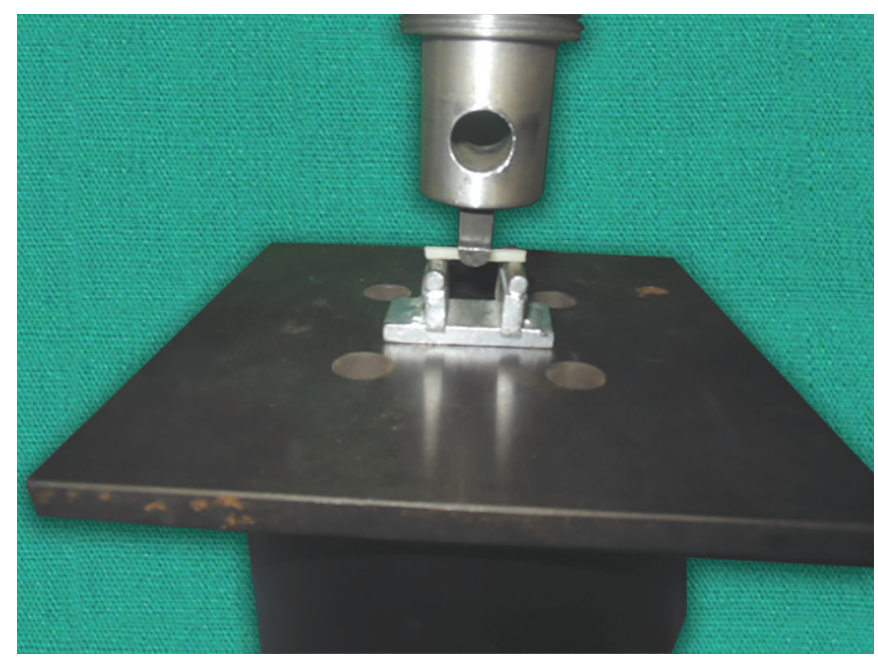

Fig. 1: Evaluation of flexural strength of sample in a universal testing machine

\section{MATERIALS AND METHODS}

A total of 80 specimens were fabricated with 20 samples of each type of restorative material namely silver amalgam (DPI alloy, Mumbai, India), GIC (Fuji Type IX, GC America), Composite (Tetric N-Ceram Ivoclar Vivadent, Mumbai, India), Cention N (Cention Ivoclar Vivadent, Mumbai, India) to test the compressive strength and flexural strength. The number of samples and their distribution in different groups for testing compressive and tensile strength were presented in (Tables 1 and 2). Cylindrical aluminum split molds of dimension $6 \pm 1 \mathrm{~mm}$ (height) $\times 4 \pm 1 \mathrm{~mm}$ (diameter) were used to fabricate 10 samples of each restorative material for testing the compressive strength and cylindrical aluminum split molds of dimension $25 \pm 1 \mathrm{~mm}$ (length) $\times 2 \pm 1 \mathrm{~mm}$ (height) $\times$ $2 \pm 1 \mathrm{~mm}$ (width) were used for fabricating samples for flexural strength.

The samples were stored in a water bath at $37 \pm 1^{\circ} \mathrm{C}$ for 24 hours before testing. The samples were tested using a UTM. The UTM was connected to a load measuring cell, which continuously recorded the load applied to the samples at a crosshead speed of $0.75 \pm 0.25 \mathrm{~mm} / \mathrm{min}$ till the samples fractured (Figs 1 and 2). One-way analysis of variance (ANOVA) (Welch) and Games-Howell post hoc test was done to determine statistically significant differences among the various restorative materials. (Graphs 1 and 2).

\section{RESULT}

Levene statistics showed that variances for compressive strength between the groups was not equal. Hence, one-way ANOVA with Welch correction was applied. One-way ANOVA (Welch) showed that there was a very high significant difference for compressive strength between different restorative materials. After one-way ANOVA (Welch), Games-Howell post hoc test was applied for

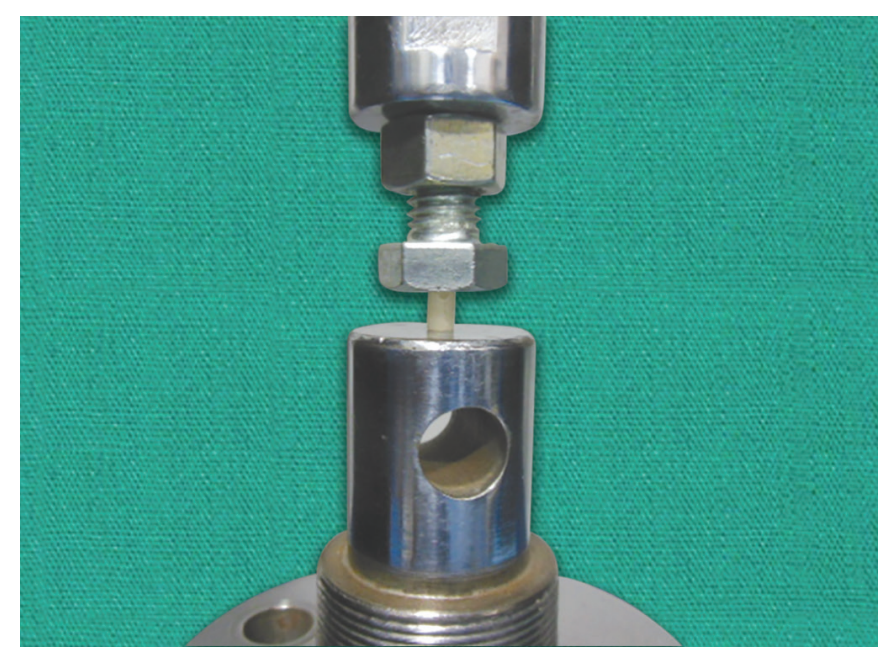

Fig. 2: Evaluation of compressive strength of sample in a universal testing machine 


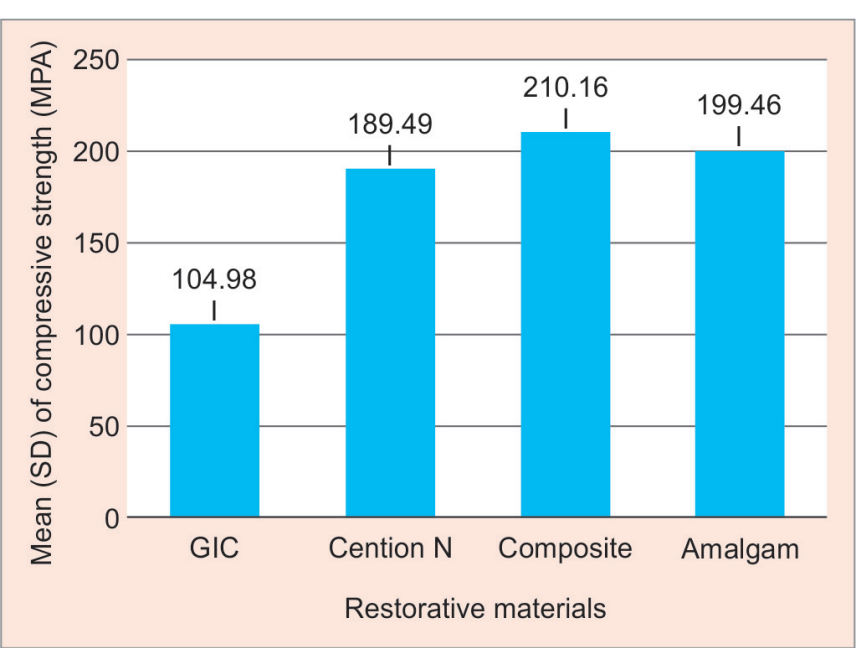

Graph 1: Mean and standard deviation of compressive strength between different restorative materials

pairwise comparison. It showed that compressive strength in composite was significantly higher than cention $\mathrm{N}$, GIC and amalgam. Compressive strength in amalgam was significantly higher than GIC and cention $\mathrm{N}$. Compressive strength in cention $\mathrm{N}$ was significantly higher than GIC. Highest compressive strength was observed in composite and lowest in GIC (Graph 1 and Table 3).

Levene statistics showed that variances for flexural strength between the groups was not equal. Hence, one-way ANOVA with Welch correction was applied. One-way ANOVA (Welch) showed that there was a very high significant difference for flexural strength between different restorative materials. After one-way ANOVA (Welch), Games-Howell post hoc test was applied for pairwise comparison. It showed that flexural strength in composite was significantly higher than cention N, GIC and amalgam. Flexural strength in cention $\mathrm{N}$ was significantly higher than GIC and amalgam. Flexural strength in GIC was significantly higher than amalgam. Highest flexural strength was observed in composite and lowest in amalgam (Graph 2 and Table 4 ).

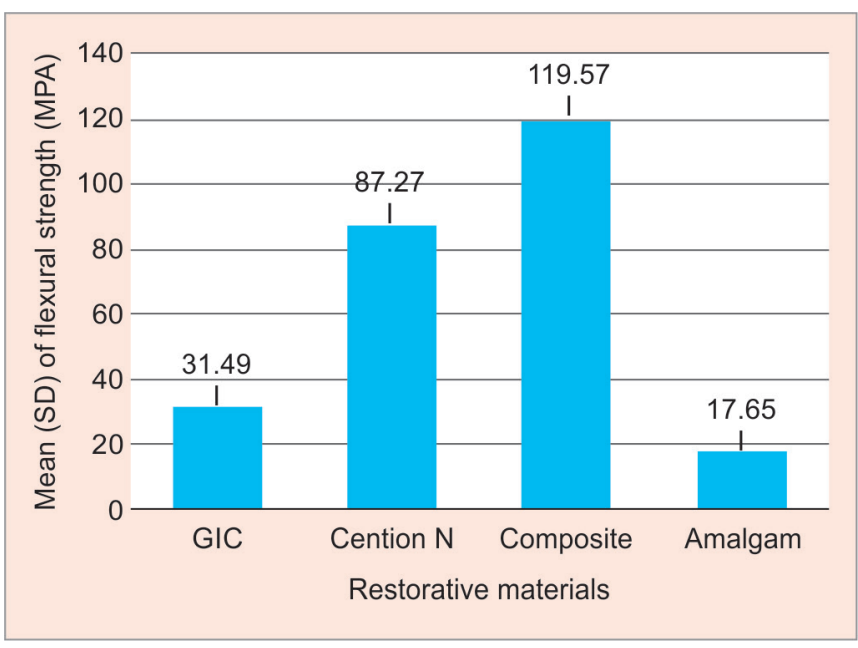

Graph 2: Mean and standard deviation of flexural strength between different restorative materials

\section{DISCUSSION}

The ultimate goal of dental restorative material is to replace the biological, functional and esthetic properties of healthy tooth structure. ${ }^{1}$ Several dental restorative materials have been used for restoration procedures like GIC, amalgam, composite since many years. ${ }^{5}$ During the last decade, due to high esthetic demands from patients, resin composites have gained popularity. ${ }^{4}$ However, like superior esthetics, strength is also one of the important criteria, as it greatly influences the selection of a restorative material according to the clinical scenario. Stronger materials resist deformation and fracture in a better way, provide more equitable stress distribution, greater stability, and greater probability of clinical success. ${ }^{3}$ Among mechanical properties, compressive strength, flexural strength and diametral tensile strength are useful and have been used widely to evaluate the clinical performance of various dental restorative materials. ${ }^{12}$ Compressive strength of restorative material is important because restorative material replace part of tooth structure and they should provide sufficient strength to resist intraoral com-

Table 1: Distribution of samples for compressive strength testing

\begin{tabular}{llll}
\hline No. of Samples & Group & Materials used & Brand name \\
\hline 10 & AC & High copper amalgam & DPI Alloy \\
10 & BC & Glass ionomer cement & Fuji Type IX \\
10 & CC & Hybrid composite & Tetric N-Ceram Ivoclar \\
10 & DC & Cention N & Vivadent \\
& & & Cention Ivoclar Vivadent \\
\hline
\end{tabular}

Table 2: Distribution of samples for tensile strength testing

\begin{tabular}{llll}
\hline No. of Samples & Group & Materials used & Brand name \\
\hline 10 & AF & High copper amalgam & DPI alloy \\
10 & BF & Glass ionomer cement & Fuji type IX \\
10 & CF & Hybrid composite & Tetric N-Ceram \\
10 & DF & Cention N & Cention ivoclar vivadent \\
\hline
\end{tabular}


Table 3: Comparison of compressive strength between different restorative materials

\begin{tabular}{llc}
\hline & & \multicolumn{1}{c}{ Compressive strength (MPa) } \\
\cline { 2 - 3 } Groups & Mean $\pm S D$ & Min-Max \\
\hline GIC $(n=10)$ & $104.98 \pm 1.18$ & $103.64-107.31$ \\
Cention N $(n=10)$ & $189.49 \pm 3.49$ & $184.54-195.82$ \\
Composite $(n=10)$ & $210.16 \pm 1.84$ & $208.33-213.27$ \\
Amalgam $(n=10)$ & $199.46 \pm 1.77$ & $196.65-202.58$ \\
One-way ANOVA (Welch) & & $F=4612.297, p=0.000$ (<0.001), Sig. diff. \\
Games-Howell post hoc test & & Composite > Amalgam > Cention N > GIC \\
\hline
\end{tabular}

Table 4: Comparison of flexural strength between different restorative materials

\begin{tabular}{llc}
\hline & \multicolumn{2}{c}{ Flexural strength $(\mathrm{MPa})$} \\
\cline { 2 - 3 } Groups & Mean $\pm S D$ & Min-Max \\
\hline GIC $(\mathrm{n}=10)$ & $31.49 \pm 0.59$ & $30.68-32.22$ \\
Cention N $(\mathrm{n}=10)$ & $87.27 \pm 1.10$ & $86.00-88.75$ \\
Composite $(\mathrm{n}=10)$ & $119.57 \pm 2.27$ & $115.65-122.22$ \\
Amalgam $(\mathrm{n}=10)$ & $17.65 \pm 1.14$ & $16.03-18.93$ \\
One-way ANOVA (Welch) & & $\mathrm{F}=11383.074, \mathrm{P}=0.000(<0.001)$, Sig. diff. \\
Games-Howell post hoc test & & Composite $>$ Cention $\mathrm{N}>$ GIC $>$ Amalgam \\
\hline
\end{tabular}

pressive and tensile forces that are produced in function and parafunction. Flexural strength is used to evaluate the strength of the material and the amount of the distortion expected under bending stress. ${ }^{2}$

The present study was conducted to compare and evaluate the mechanical properties of cention $\mathrm{N}$ with other basic restorative materials namely GIC, silver amalgam and resin composite. In this study, compressive strength in composite was significantly higher than cention N, GIC and slightly higher than that of silver amalgam, which was similar to the results of a study done by Cohen et al. ${ }^{13}$ In their study it was stated that this could be due to the micromechanical bonding (Monoblock effect) of resins to the tooth structure and resin composites behaving like stress breakers as well as complete curing of material with dual cure technology. ${ }^{14-16}$ Results of our study were also consistent with the results of study done by Cho et al. ${ }^{17}$ and Agrawal et al..$^{18}$ who found that some resin composites exhibited compressive strengths more than that of silver amalgam and could be used as alternatives to silver amalgam. Compressive strength in cention $\mathrm{N}$ was significantly higher than GIC but lesser than composite. The reason may be because it does not have a comparable micromechanical bond to the tooth structure. The company recommends that if cention $\mathrm{N}$ is restored without using an adhesive, tooth preparation is done like for conventional amalgam i.e., with retentive features. Cention $\mathrm{N}$ is supplied as two-part powder/liquid systems unlike composite, and therefore variation in the powder: liquid ratio may influence the final result. Compressive strength in silver amalgam was significantly higher than GIC but lower than composite. This result is in accordance with the results of the study by Cho et al. ${ }^{17}$ They stated that this could be due the fact that the modulus of elasticity of composites is approximately one third the modulus of elasticity of amalgam; that is, amalgams are three times more rigid. It showed that flexural strength in composite was significantly higher than cention $\mathrm{N}$, GIC and amalgam. Flexural strength in cention $\mathrm{N}$ was significantly higher than GIC and amalgam. Flexural strength in GIC was significantly higher than amalgam. Highest flexural strength was observed in composite and lowest in amalgam. The high flexural strength of composite was reported to be higher due to the higher filler concentration. In the present study the better performance of composite and Cention $\mathrm{N}$ could be attributed to the composition of monomer used (UDMA). Astudy done by Erik Asmussen, Anne Peutzfeldt on the influence of UDMA, BisGMA and TEGDMA on mechanical properties of experimental resin composites showed that the monomer containing BisGMA or TEGDMA substituted by UDMA resulted in an increase flexural strength, and that substitution of BisGMA by TEGDMA reduced the flexural strength. The Flexural strength value for amalgam was the least when compared to all the materials tested. This could be due the fact that the modulus of elasticity of composites is approximately one-third the modulus of elasticity of amalgam. ${ }^{1}$

At last we can say that cention $\mathrm{N}$ can be used in various restorative procedures in daily dental practice as a basic filling material along with tooth matching ability, it has 
good mechanical properties and unlike composite its economical to patients. Despite such standardization, these data demonstrate variation, as limitations still exist when trying to extrapolate these results to the clinical performance of materials Also the oral cavity condition difficulties like saliva, visibility issues, operator handling of material etc. which could play an important role in curing and setting of the material which would influence the strength of the material greatly. Selection of restorative material must include the understanding of materials properties and no one material may be considered ideal and capable of truly replacing lost tooth structure. The clinicians should have a clear knowledge of the mechanical properties of the materials to obtain the best clinical outcome.

\section{CONCLUSION}

Within the limitations of this in vitro study, the following conclusions were drawn:

Composite had the highest compressive strength and flexural strength of the four materials tested in the study.

The compressive strength of cention $\mathrm{N}$ was less than composite and silver amalgam but higher than GIC. Flexural strength of Cention $\mathrm{N}$ was less than composite but higher than silver amalgam and GIC.

The strength of GIC in the present study was found to be inadequate to be used as a restorative material in a stress bearing area as its compressive strength in comparison to composite and silver amalgam is less.

\section{REFERENCES}

1. Deepika K, Hegde M, Hegde P, Bhandary S. An evalution of compressive strength of newer nanocomposite: An in vitro study. J Conserv Dent. 2011;14(1):36-39.

2. Sakaguchi R, Powers J. Craig's Restorative Dental Materials. 13th edition, Philadalphia

3. Shivrayan A, Kumar G. Comparative study of mechanical properties of direct core build-up materials. Contemp Clin Dent. 2015;6(1):16-20.

4. Yüzügüllü B, Çiftçi Y, Saygili G, Canay. Diametral tensile and compressive strengths of several types of core materials. J Prosthodont. 2008;17(2):102-107.
5. Jayanthi N, Vinod V. Comparative evaluation of compressive strength and flexural strength of conventional core materials with nanohybrid composite resin core material an in vitro study. J Indian Prosthodont Soc. 2013;13(3):281-289.

6. Anusavice KJA. Harrison RH. Philips' Science of Dental Materials 11th edition. Saunders, Philadelphia, 1992. p. 370-374.

7. Affairs AC. Dental amalgam: Update on safety concerns. J Am Dent Assoc. 1998 Apr 1;129(4):494-503.

8. Parolia A, Adhauliya N, de Moraes Porto IC, Mala K. A comparative evaluation of microleakage around class $\mathrm{V}$ cavities restored with different tooth colored restorative materials. Oral Health Dent Manag. 2014;13(1):120-126.

9. Zanata RL, Navarro MF, Ishikiriama A, da Silva e Souza Júnior $\mathrm{MH}$, Delazari RC. Bond strength between resin composite and etched and non-etched glass ionomer. Braz Dent J. 1997; 8(2):73-78.

10. Cardoso M V, Yoshida Y, van Meerbeek B. Adhesion to tooth enamel and dentin - a view on the latest technology and future perspectives. Chapter 3 in: Roulet J-F, Kappert H F. Statements: Diagnostics and therapy in dental medicine today and in the future. 2009. Quintessence.

11. Cention N retrieved from- http:/ / www.ivoclarvivadent.in/ en-in/p/all/cention-n.

12. Yamazaki T, Schricker SR, Brantley WA, Culbertson BM, Johnston W. Viscoelastic behavior and fracture toughness of six glass-ionomer cements. J Prosthet Dent. 2006;96(4): 266-272.

13. Cohen BI, Pagnillo MK, Deutsch AS, Systems ED, Hackensack $\mathrm{S}$. Fracture strengths of three core restorative materials supported with or without a prefabricated split-shank post. J Prosthet Dent. 1997;78(6):560-565.

14. Gateau P, Sabek M, Dailey B. In vitro fatigue resistance of glass ionomer cements used in post-and-core applications.J Prosthet Dent. 2001;86(2):149-155.

15. Sidoli GE, King PA,Setchell DJ . An in vitro evaluation of a carbon fiber-based post and core system. J Prosthet Dent.1997;78(1):5-9.

16. Shaini FJ, Fleming GJP, Shortall ACC, Marquis PM. A comparison of the mechanical properties of a gallium-based alloy with a spherical high-copper amalgam. Dent Mater. 2001;17(2):142-148.

17. Cho GC, Kaneko LM, Donovan TE, White SN. Diametral and compressive strength of dental core materials. J Prosthet Dent 1999;82:272-276.

18. Agrawal A, Mala K. An in vitro comparative evaluation of physical properties of four different types of core materials. J Conserv Dent. 2014;17(3):230-233. 